\title{
Disconnection analysis of CA3 and DG in mediating encoding but not retrieval in a spatial maze learning task
}

\author{
Taylor Jerman, Raymond P. Kesner, ${ }^{1}$ and Michael R. Hunsaker \\ Department of Psychology, University of Utah, Salt Lake City, Utah 84112, USA
}

\begin{abstract}
The dentate gyrus (DG) subregion of the hippocampus has been shown to be involved in encoding but not retrieval in a spatial maze task (modified Hebb-Williams maze). The first experiment in this study examined whether a lesion to the CA3 would contribute to a similar encoding deficit. A DG group was included in order to replicate previous results. Relative to controls, animals receiving CA3 lesions were impaired in encoding, not retrieval, on the modified Hebb-Williams maze-similar to a group that received DG lesions. This suggests the possibility that CA3 and DG are working together to mediate encoding processes. The second experiment in this study was designed to test the interaction between CA3 and DG using a disconnection paradigm. Animals with contralateral lesions (CA3 lesioned in one hemisphere, DG lesioned in the other hemisphere) showed a significant disruption effect on encoding, but not retrieval, when compared with animals with ipsilateral lesions (CA3 and DG lesioned in the same hemisphere, leaving the other hemisphere intact). This suggests an interaction between CA3 and DG in supporting encoding but not retrieval processes in a spatial maze learning task.
\end{abstract}

\begin{abstract}
The CA3 subregion of the hippocampus appears to be involved in a number of processes including the encoding of new information, pattern completion, short-term memory, and pattern associations and may participate in pattern separation (Kesner et al. 2004). One of the mnemonic processes suggested for CA3 is the encoding of new information, originally suggested by Marr (1971). Marr proposed that the CA3 network in the hippocampus should be capable of a rapid formation of simple representations, based on modifiable synaptic connections among its neurons. Recent experimental evidence supports this theoretical hypothesis. Lee and Kesner (2002) manipulated the N-methyl-Daspartate (NMDA) receptors in CA3 by injecting APV (amino-5phosphonovaleric acid, a pharmacological blocker for the NMDA receptors) selectively into CA3 in a delayed non-matching-toplace (DNMP) task. They found that rats injected with APV were not impaired in performing the DNMP task in a familiar environment in which they had been trained. However, they were significantly impaired in initially performing the task normally when the same task was carried out in a completely novel environment (i.e., a novel testing room). APV injected in adjacent hippocampal subregions (e.g., DG or CA1) did not produce such deficits in the novel environment. Similar results were obtained with CA3-specific neurotoxic lesions (Lee and Kesner 2003). Nakazawa et al. (2003) reported similar findings with a mouse strain in which the function of CA3 NMDA receptors was disrupted. These mutant mice were impaired in learning a novel platform location in a modified water maze task, whereas they were normal in finding familiar platform locations. In sum, these results strongly suggest that rapid plastic changes in the CA3 network are essential in encoding novel information quickly into the hippocampal memory system and that NMDA receptor-mediated plasticity mechanisms appear to play a significant role in the process.

It is important to note that the CA3 region is also important
\end{abstract}

'Corresponding author.

E-mail rpkesner@behsci.utah.edu; fax (801) 581-5841.

Article is online at http://www.learnmem.org/cgi/doi/10.1101//m.246906. for encoding information that may require more than one trial. For example, learning and/or encoding of an object-place or odor-place paired-associate task required a large number of trials but was impaired following CA3 lesions (Gilbert and Kesner 2003). Learning to navigate through a Hebb-Williams maze requires multiple trials, and the encoding of the information has been shown to be disrupted by DG lesions without affecting retrieval. However, a lesion of the perforant path input into CA3 has been shown to disrupt retrieval and leave encoding intact (Lee and Kesner 2004b). Because lesions of the mossy fiber input into CA3 produce an encoding deficit, whereas lesions of the perforant path input into CA3 produce a retrieval deficit, it is not clear what the effects of a CA3 lesion would be on encoding and retrieval. Thus, the first major aim of the present study was to determine whether the CA3 subregion of the hippocampus supports either an encoding or a retrieval process or both an encoding and retrieval process. This aim will be accomplished by making separate lesions of CA3 and DG followed by tests on the Hebb-Williams maze. The Hebb-Williams maze was selected because it is a spatial task that has been shown to be sensitive to hippocampal dysfunction, can be learned quickly, and requires serial organization of specific response patterns to achieve optimal performance on the maze. During acquisition of the HebbWilliams maze, it is assumed that the encoding phase dominates as the rat learns the maze within a day of testing, whereas the retrieval phase dominates as the rat initially runs on the maze after a 24-h delay during which some consolidation may have occurred. It is also assumed that encoding encompasses spatial pattern separation processes in conjunction with associative processes and representations within short-term memory. Although there is likely to be some retrieval from short-term memory that may occur during acquisition, it is assumed that encoding processes override retrieval processes and thus dominate within the first 10 trials during Day 1. Furthermore, it is assumed that retrieval after $24 \mathrm{~h}$ encompasses associative processes as well as representations within intermediate-term memory. Although there is likely to be some encoding that may also occur during retrieval, it is assumed to be overridden and dominated by retrieval processes within the first five trials of Day 2 . The purpose 
of these experiments was to test computational models using behavioral paradigms; therefore, the terms "encoding" and "retrieval" are used and are defined as they are.

The second aim addresses the issue that if there were an encoding, but not a retrieval, deficit for the DG and CA3 in the first experiment, could there be an interaction in terms of the encoding processes between the DG and the CA3? This cooperation between DG and CA3 could theoretically derive from the observation that the DG granular neurons project to CA3 pyramidal neurons via mossy fiber projections forming the primary output of the DG. Based on characteristics of the mossy fiber system, Rolls (1996) suggests that pattern separation may be a function of the DG and its mossy fiber projections to CA3 and thus may facilitate the encoding of spatial information via an interaction between the DG and CA3. However, recent studies have shown that the functions of these two hippocampal subregions can be dissociated using behavioral tasks. For example, Gilbert and Kesner (2003) demonstrated that DG lesioned animals were able to learn object-place and odor-place pairedassociate tasks as quickly as controls. However, rats with CA3 lesions showed significant learning impairments on both tasks. Furthermore, a lesion of the perforant path input into the CA3 also disrupted object-place paired-associate learning (I. Lee, P.E. Gilbert, and R.P. Kesner, unpubl.). These results could indicate that the CA3, but not the DG, subregion is involved in associative learning. Furthermore, DG, but not CA3, lesioned rats produce a deficit in the acquisition of the standard version water maze (Sutherland et al. 1983; Nanry et al. 1989; Xavier et al. 1999; Lassalle et al. 2000).

These dissociations arise because possible dissociations between DG and CA3 are primarily due to the CA3 subregion of the hippocampus having two major inputs with a direct connection from the DG via the mossy fibers and a direct input from the perforant path that bypasses the DG. An alternate explanation is possible since a lesion of the perforant path disrupts retrieval but not encoding (Lee and Kesner, 2004b). The differences between DG and CA3 in the above-mentioned tasks could be simply a function of differential intrinsic processing of similar spatial information. As another example, Gilbert et al. (2001) and Gilbert and Kesner (2006) tested rats with DG or CA3 lesions using a paradigm that measured one-trial short-term memory for spatial location information as a function of spatial similarity between two spatial locations. The results showed that rats with DG lesions were significantly impaired at short spatial separations; however, the performance of the DG lesioned rats increased as a function of increased spatial separation between the correct object and the foil on the choice phases. In contrast, CA3 lesioned rats were impaired for all spatial separations, suggesting a disruption of a short-term memory process subserved by CA3 as an intrinsic contribution to the spatial pattern separation task. Therefore, there is no guarantee that a potential cooperation between DG and CA3 in encoding information in the HebbWilliams maze is due to the direct connection between these two regions. To examine this issue a disconnection study was carried out using an ipsilateral lesion (DG and CA3 lesion on one side only) group vs. a contralateral lesion (DG on one side and CA3 on the other side) group. It is assumed the right and left hemispheres operate in parallel. Crossed lesions (i.e., unilateral lesions in contralateral hemispheres), therefore, would disrupt communication within each of the two hemispheres, thus disconnecting the two brain regions. If the DG and CA3 subregions of the hippocampus interact, then crossed lesioned rats should be markedly impaired compared with animals with lesions on the same side. If these two regions do not interact, then crossed lesions would not produce a deficit, suggesting that each subregion produces a deficit in encoding for different reasons, such as spatial pattern separation problems vs. associative or short-term memory problems.

\section{Results}

\section{Histological analysis}

Axon-sparing, subregion-specific lesions of the dorsal hippocampus were made with specific neurotoxins. Colchicine $(2.5 \mathrm{mg} /$ $\mathrm{mL}, 20.0 \mu \mathrm{l} / \mathrm{hr}$ ) was used for the bilateral DG lesions, and ibotenic acid $(6.0 \mathrm{mg} / \mathrm{mL}, 6.0 \mu \mathrm{l} / \mathrm{hr})$ was used for the bilateral CA3 lesions. A quantitative analysis revealed that lesions of the dentate gyrus (Fig. 1A) were $94.63 \pm 1.69 \%$ complete $(\% \pm$ SEM), with no visible damage to CA3 and, in one animal, $2.5 \%$ damage to the overlying CA1 subregion. A quantitative analysis revealed that lesions of the CA3 (Fig. 1B) were $\sim 88.69 \pm 1.87 \%$ complete, with sparing at the CA2 aspect of CA3 and no cortical damage. There were few instances of small damage to CA1 or to the dentate gyrus of less than $2 \%$. In all lesions there was some sparing mostly at the septal pole of the hippocampus, but the lesion was quantified from 1.8 to $4.3 \mathrm{~mm}$ posterior to bregma. There was no observed damage to the ventral half of the hippocampus ( $>4.5$ $\mathrm{mm}$ posterior to bregma).

Figure 1C shows a representative example of a lesion of DG on the left side and a CA3 lesion on the right side. The damage to the dentate gyrus is comparable with the bilateral DG lesion on the left side and intact except for some small damage on the ventral blade of the dentate gyrus on the contralateral side $(94.67 \pm 2.64 \%)$. The damage to the CA3 is complete on the right side with no observable damage on the contralateral side $(91.83 \pm 2.87 \%)$. The quantitative analyses of damage to dentate gyrus and CA3 are similar to what has been described above for the dentate gyrus and CA3 lesions. The observation of an intact CA3 on the contralateral side to the CA3 lesion suggests the possibility that the CA3 lesion did not produce significant degeneration in the contralateral side. Figure 1D shows a representative example of an ipsilateral lesion of dentate gyrus and CA3 on one side. Note that the damage in this case also involved CA1 in all animals, so that the lesion can be characterized as a uni-
A

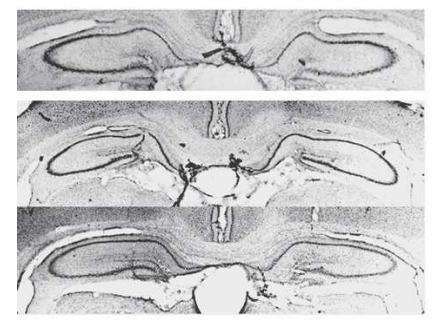

C

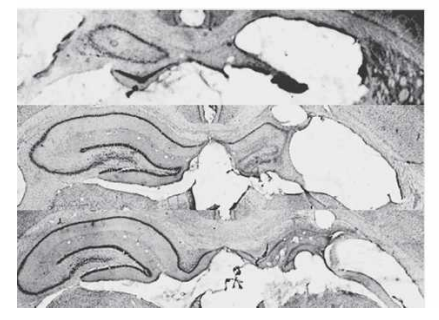

B

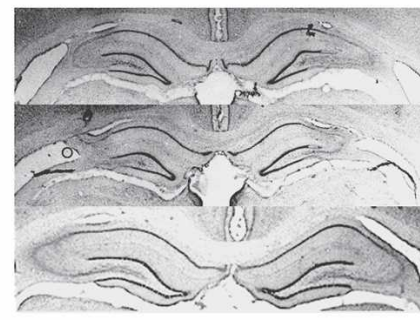

D

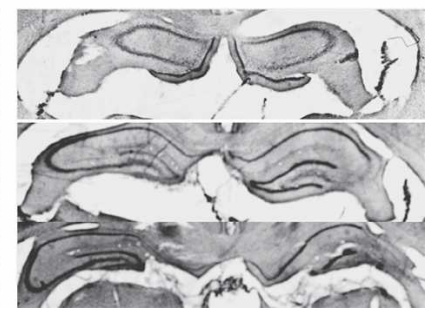

Figure 1. Representative photomicrographs $(12 \times)$ of lesions to hippocampal subregions. (A) Dentate gyrus colchicine-induced lesion. (B) CA3 ibotenic acid lesion. (C) Contralateral lesion of dentate gyrus (left) and CA3 (right). (D) Ipsilateral lesion of dentate gyrus and CA3 resulting in full unilateral hippocampal ablation. See Results for quantitative analysis. 
lateral dorsal hippocampus lesion with no hippocampus damage on the opposite side to the lesion (total hippocampal damage $94.67 \pm 2.68 \%)$.

\section{Behavioral analysis}

The question posed in the first experiment was whether lesions of the DG or CA3 would disrupt encoding without affecting retrieval. The effects of these lesions on performance in the HebbWilliams maze in terms of mean number of errors per trial shown as grouped in five-trial blocks for $3 \mathrm{~d}$ are shown in Figure 2. The data indicate that the CA3 group appears to make more errors than the DG and control groups. A two-way ANOVA with groups as the between variable and blocks of five trials as the within variable revealed that there was a significant group effect $\left[F_{(2,29)}=4.45 ; P<0.021\right]$ and a significant days effect $\left[F_{(2,29)}=204.97 ; P<0.0001\right]$. A Tukey HSD paired-comparison test on the group effect revealed that the CA3 lesioned rats displayed significantly more errors than the control group and the DG group $(P<0.05)$.

For a detailed analysis of the encoding and retrieval processes, a separate index was developed for each process. It is assumed that encoding and retrieval processes interact on a trialby-trial basis in any behavioral task. Thus, it is difficult to discriminate between the contributions of encoding and retrieval for individual trials using a behavioral analysis. On the modified Hebb-Williams maze, however, compared with the experimental group, the control group exhibited measurably improved performance for Days 1 and 2. Thus, encoding and retrieval were given operational definitions based on calculations of trial errors. Encoding index was calculated within a day by subtracting the average of the last five trials of Day 1 (D1b) from the average of the first five trials of Day 1 (D1a). Retrieval index was calculated by subtracting the average of the first five trials of Day 2 (D2a) from the average of the last five trials of Day 1 (D1b). Therefore, a larger encoding score (D1a - D1b) indicated more effective encoding during Day 1, and a larger retrieval score (D1b - D2a) indicated more effective retrieval between Days 1 and 2 . The assumption made in this study was that the encoding of information predominated during testing on Day 1 and the retrieval of information predominated during testing on Day 2, $24 \mathrm{~h}$ later.

The effects of CA3 and DG lesions on encoding are shown in Figure $3 \mathrm{~A}$ and indicate that, relative to controls, there is a reduction in the mean encoding index for both the CA3 and DG lesioned rats. A one-way ANOVA for groups revealed that there was a significant group effect $\left[F_{(2,31)}=6.3 ; P=0.005\right]$. A Tukey HSD

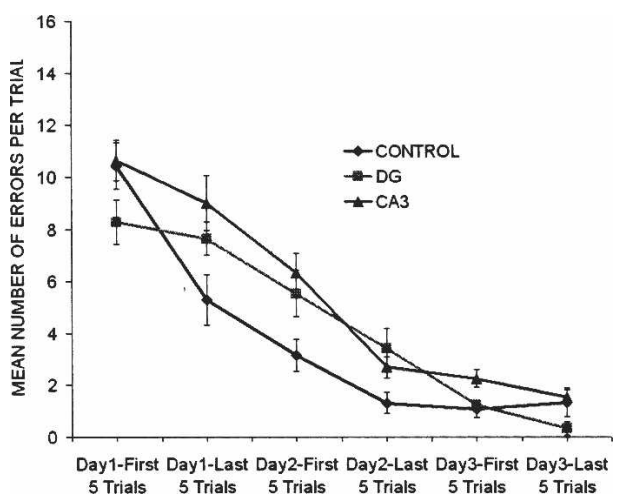

Figure 2. Mean number of errors per trial were grouped in five-trial blocks across a total of $3 \mathrm{~d}$ of acquisition in the Hebb-Williams maze for the control, CA3, and dentate gyrus (DG) groups. The CA3 lesions group made more errors than DG or control groups as determined by a Tukey HSD paired comparison $(P<0.05)$.
A

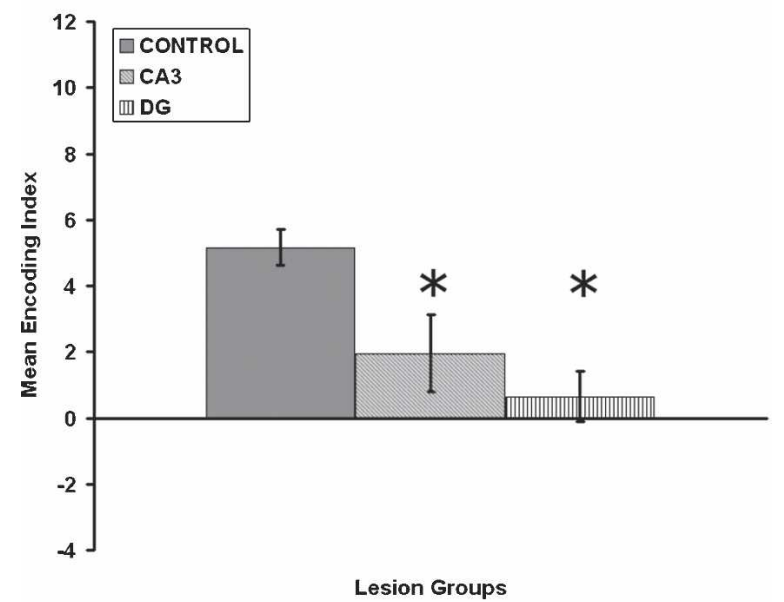

B

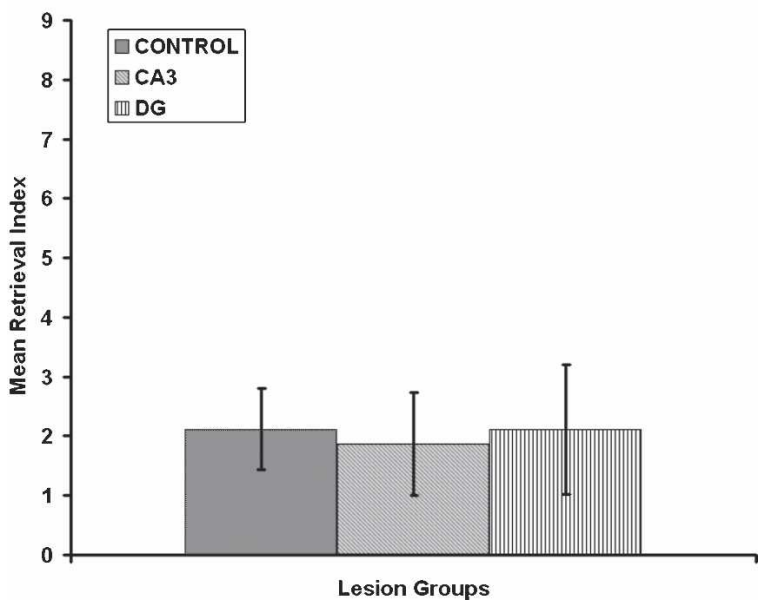

Figure 3. ( $A$ ) Mean encoding index in the Hebb-Williams maze for the control, CA3, and dentate gyrus (DG) groups. (B) Mean retrieval index in the Hebb-Williams maze for the control, CA3, and dentate gyrus (DG) groups. The results show that CA3 and DG groups disrupt encoding but have no deleterious effects on retrieval. Asterisks $\left(^{*}\right)$ correspond to significant differences from controls $(P<0.01)$ as determined by Tukey HSD paired-comparison test.

paired-comparison test on the group effect revealed that the CA3 and DG lesioned rats displayed a significantly lower encoding index than the control group $(P<0.01)$.

The effects of CA3 and DG lesions on retrieval are shown in Figure 3B and indicate that, relative to controls, there is comparable performance for the retrieval index for both the CA3 and DG lesioned rats. A one-way ANOVA for groups revealed that there was no significant group effect $\left[F_{(2,31)}=0.03 ; P>0.05\right]$. Thus, both CA3 and DG lesioned rats display impairments for encoding, but not retrieval of spatial information.

The question posed in the second experiment was whether there is an interaction in terms of the activation of encoding processes between the DG and the CA3 without affecting retrieval. The effects of control, ipsilateral, and contralateral lesions on performance in the Hebb-Williams maze in terms of mean number of errors per trial grouped in 5-trial blocks for $3 \mathrm{~d}$ are shown in Figure 4 . The data indicate that there were no differences among the groups and that all three groups improved so that by Day 3 they were not making many errors. A two-way ANOVA with groups as the between variable and blocks of five trials as the within variable revealed that there was no significant 


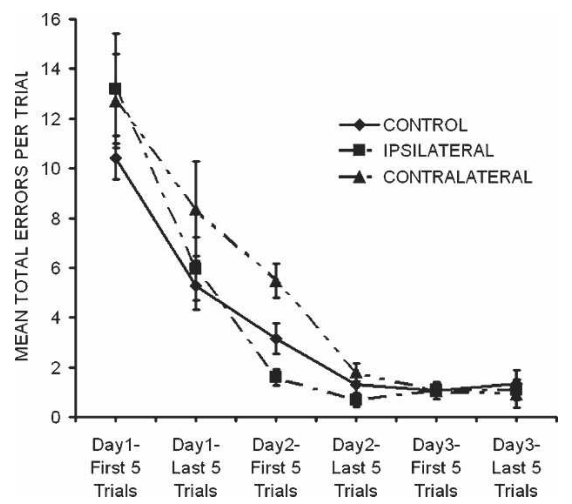

Figure 4. Mean number of errors per trial were grouped in five-trial blocks across a total of $3 \mathrm{~d}$ of acquisition in the Hebb-Williams maze for the contralateral and ipsilateral lesion groups. The results show that the contralateral, but not ipsilateral, treatment disrupted encoding, but neither group had any deleterious effects on retrieval.

group effect $\left[F_{(2,24)}=2.893 ; P>0.05\right]$ but a significant days effect $\left[F_{(2,24)}=189.753 ; P<0.0001\right]$ and no significant interaction $\left[F_{(2,24)}=1.858, P>0.05\right]$. Since the effect of group approached significance $(P=0.077)$, a Tukey HSD paired-comparison test was performed on lesion groups. It revealed that contralateral lesioned animals were not significantly impaired relative to control or ipsilaterally lesioned animals $(P>0.05)$.

The effects of ipsilateral and contralateral DG-CA3 lesions on encoding are shown in Figure 5A and indicate that relative to controls there is a reduction in the mean encoding index for the contralateral, but not the ipsilateral, rats. A one-way ANOVA for groups revealed that there was a significant group effect $\left[F_{(2,22)}=7.15 ; P=0.004\right]$. A Tukey HSD paired-comparison test on the group effect revealed that the contralateral lesioned rats displayed a significantly lower encoding index than the control and ipsilateral groups $(P<0.01)$.

The effects of ipsilateral and contralateral DG-CA3 lesions on retrieval are shown in Figure 5B and indicate that relative to controls there is comparable performance for the retrieval index for both the CA3 and DG lesioned rats. A one-way ANOVA for groups revealed that there was no significant group effect $\left[F_{(2,22)}=1.27 ; P>0.05\right]$. Thus, contralateral, but not ipsilateral, lesioned rats displayed impairment in encoding, but neither group displayed impairment in retrieval of spatial information.

\section{Discussion}

In the Hebb-Williams learning task one can measure improvement in performance within a day reflecting the operation of encoding of new information based in part on short-term memory representations. One can also measure improvement in performance between days reflecting the operation of retrieval of information based on intermediate-term memory representations mediated by synaptic consolidation and/or access to stored information. It is recognized that the separation of encoding from retrieval processes is extremely difficult. Therefore, it is assumed that during acquisition within a day, there will be a greater involvement of encoding than retrieval processes, and that during retention across days, there will be a greater involvement of retrieval than encoding processes. It is also assumed that encoding encompasses spatial pattern separation processes in conjunction with associative processes and representations within short-term memory. Although there is likely to be some retrieval from short-term memory that may also occur during acquisition, it is assumed not to be the dominant factor governing performance within the first 10 trials on Day 1. Furthermore, it is assumed that retrieval $24 \mathrm{~h}$ later encompasses associative processes as well as representations within intermediate-term memory. Although there is likely to be some encoding that may also occur during retrieval, it is assumed not to be the most critical determinant of performance within the first five trials on Day 2.

It is, therefore of interest that our results indicate that both DG and CA3 lesions disrupted encoding, but not retrieval, even though CA3 lesions did produce more errors during acquisition than DG lesions. The DG data represent a replication of previous research where DG lesions disrupted encoding, but not retrieval, on the Hebb-Williams maze (Lee and Kesner 2004b). The DG lesion data are also consistent with the results of Lassalle et al. (2000), who showed that in a water-maze learning task, lesions of the mossy fibers disrupted encoding but not retrieval. The CA3 lesion data are also consistent with the idea that short-term encoding of new information is mediated by the CA3. Other studies with alteration of CA3 function support this idea (Lee and Kesner 2002, 2003, 2004a; Nakazawa et al. 2003). Thus, the data suggest

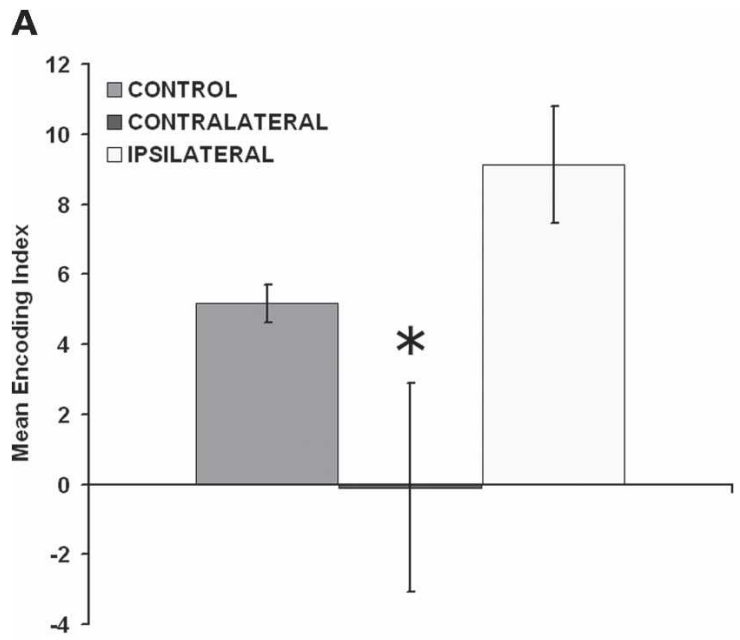

Lesion Groups

B

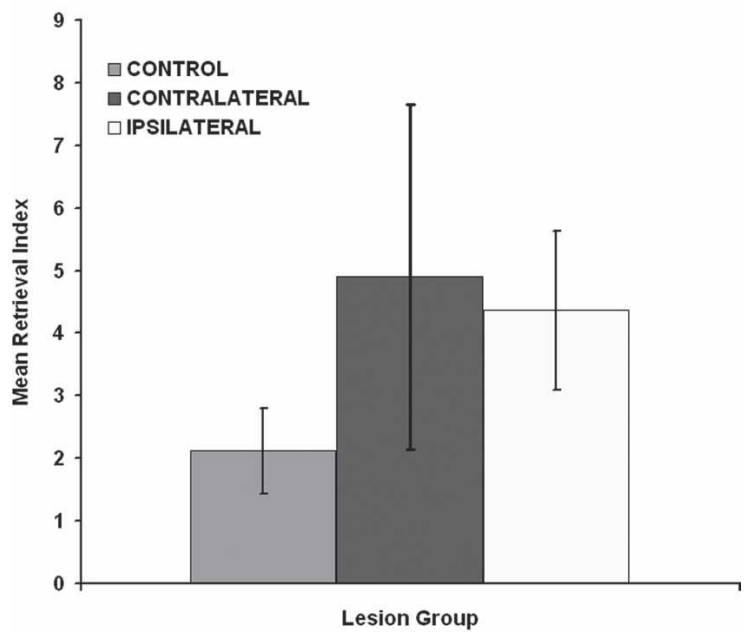

Figure 5. (A) Mean encoding index in the Hebb-Williams maze for the control, contralateral, and ipsilateral lesions groups. (B) Mean retrieval index in the Hebb-Williams maze for the control, contralateral, and ipsilateral lesions groups. Asterisks $\left({ }^{*}\right)$ correspond to significant differences from controls $(P<0.01)$ as determined by the Tukey HSD pairedcomparison test. 
a possible cooperation between the DG and CA3 in encoding of information in a Hebb-Williams maze, in that there is a deficit in encoding of information following either DG or CA3 lesions.

Our data appear to be in conflict with the results of Lee and Kesner (2004b) in which a lesion to the perforant path caused a deficit in retrieval but no effect for encoding. There is the possibility that the lesion to the perforant path may have disrupted the Schaffer collateral output from CA3 into CA1 as well as the perforant path inputs from the entorhinal cortex, and perhaps some of CA1 as well. Since the Lee and Kesner paper was published (2004b), Hebb-Williams maze data for CA1 lesioned animals from our laboratory have shown that animals with lesions to dorsal CA1 show no deficit in encoding but a significant deficit in retrieval relative to a control group (Vago and Kesner 2005), identical results to those seen in perforant path lesioned animals. If the Schaffer collateral input into CA1 were disrupted by the perforant path lesion performed by Lee and Kesner (2004b), then the effects seen in that study would be more indicative of a CA1 effect than a CA3 effect if taken in light of the results of the present study. This would be more consistent with our results indicating that a lesion to CA3 does not result in a deficit for retrieval of information-only encoding.

The results of the second experiment indicate that rats with an ipsilateral lesion of both DG and CA3 do not have a deficit in either encoding or retrieval in the Hebb-Williams maze. The fact that the lesion involved CA1 in addition to DG and CA3 does not invalidate the results since there were no deficits with the larger unilateral lesion. In contrast, rats with a DG lesion on one side of the brain and a CA3 lesion on the other side of the brain disrupted encoding, but not retrieval, of information on the HebbWilliams maze. Thus, the DG and CA3 interact in supporting encoding of spatial information in the present task. There is always the possibility that following a DG lesion on one side and a CA3 lesion on the other side that there would be a significant amount of degeneration of CA3 following the DG lesion, and in effect creating a bilateral CA3 lesion. The same could occur for the DG following a CA3 lesion. Against this argument is the fact that, in the histology presented, there is no clear loss of the contralateral CA3 following the DG lesion and no loss of the contralateral DG following the CA3 lesion (cf. Jerman et al. [2005] for data concerning subregional specificity of CA3 and DG lesions). Thus, the data suggest that the DG and CA3 cooperate and interact with each other in the encoding of new spatial information in the Hebb-Williams maze without affecting retrieval. This cooperation can derive from the observation that the DG granular neurons project to CA3 pyramidal neurons via mossy fiber projections that form the primary output of the DG. Based on characteristics of the mossy fiber system, Rolls (1996) suggested that pattern separation may be a function of the DG and its mossy fiber projections to CA3. Pattern separation is described as a mechanism for separating partially overlapping patterns of activation so that one pattern may be retrieved as separate from any other pattern. Rolls' model proposes that pattern separation is facilitated by sparse connections in the mossy-fiber system, which connects DG granular cells to CA3 pyramidal neurons. The separation of patterns is accomplished based on the low probability that any two CA3 neurons will receive mossy-fiber input synapses from a similar subset of DG cells. The mossy fiber inputs to CA3 from DG are suggested to be essential during learning and may influence which CA3 neurons will fire based on the distributed activity in the DG. The cells of the DG are suggested to act as a competitive learning network with Hebb-like modifiability to reduce redundancy and produce sparse, orthogonal outputs. Therefore, pattern separation may facilitate the encoding of spatial information via an interaction between the DG and CA3 learning. To examine the contribution of the DG to spatial pattern separation, Gilbert et al. (2001) tested rats with DG lesions using a paradigm that measured one-trial short-term memory for spatial location information as a function of spatial similarity between two spatial locations. The results showed that rats with DG lesions were significantly impaired at short spatial separations; however, the performance of the DG lesioned rats increased as a function of increased spatial separation between the correct object and the foil on the choice phases. The performance of rats with DG lesioned matched that of controls at the largest spatial separation. The graded nature of the impairment and the significant linear increase in performance as a function of increased separation illustrate the deficit in pattern separation produced by DG lesions. Additional evidence comes from a recent study (Goodrich-Hunsaker et al. 2005) using a modified version of an exploratory paradigm developed by Poucet (1989) in which rats with DG lesions and controls were tested on tasks involving a metric spatial manipulation. The results showed that DG lesions impaired detection of a metric distance change in that rats with DG lesions spent significantly less time exploring the two objects that were displaced.

The results of both experiments provide for empirical validation of the role of DG in spatial pattern separation. Support for a role of CA3 in pattern separation comes from a study by Tanila (1999), who showed that CA3 place cells were able to maintain distinct representations of two visually identical environments and selectively reactivate either one of the representation patterns depending on the experience of the rat. Also, Leutgeb et al. (2004) recently showed that when rats experienced a completely different environment, CA3 place cells developed orthogonal representations of those different environments by changing their firing rates between the two environments, whereas CA1 place cells maintained similar responses. In a different study, Vazdarjanova and Guzowski (2004) placed rats in two different environments separated by $\sim 30 \mathrm{~min}$. The two environments differed greatly in that different objects were located in each room. The authors were able to monitor the time course of activations of ensembles of neurons in both CA3 and CA1, using a new immediate-early gene-based brain-imaging method (ArC/H1a catFISH). When the two environments were significantly different, CA3 neurons exhibited lower overlap in their activity between the two environments than CA1 neurons. Thus, CA3 may represent different environments relatively orthogonally. It is likely that the actual pattern separation may be performed as a result of the operation of the dentate granule cells as a competitive net and the nature of the mossy fiber connections to CA3 cells.

In summary, the data suggest that the DG and CA3 cooperate and interact with each other in the encoding of new spatial information in the Hebb-Williams maze without affecting retrieval.

\section{Materials and Methods}

\section{Subjects}

Forty-eight male Long-Evans rats, $\sim 2$ mo old and weighing $\sim 300$ $\mathrm{g}$ at the start of the experiment, were used as subjects. Each rat was individually housed in standard plastic rodent cages located in a colony room. The colony room was maintained on a 12 -h light/12-h dark cycle. All rats had unlimited access to water but were initially food deprived to $85 \%-90 \%$ of free-feeding weight. All testing was conducted during the light portion of the light/ dark cycle.

\section{Surgery}

In the first experiment rats were randomly assigned to receive neurotoxic lesions into CA3 $(n=13)$ and DG $(n=8)$ and vehicle injections into CA3 $(n=7)$ and into DG $(n=6)$. Ibotenic acid (6 
$\mathrm{mg} / \mathrm{mL}$ ) was used to destroy pyramidal cells in CA3, and colchicine $(2.5 \mathrm{mg} / \mathrm{mL})$ was used to destroy granule cells in the DG as it has been shown to selectively lesion DG granule cells (Goldschmidt and Steward 1982; Mundy and Tilson 1990). In the second experiment, ipsilateral lesioned animals $(n=6)$ received unilateral dorsal DG and CA3 lesions (i.e., DG and CA3 were both lesioned on the same side of the brain). Within this ipsilateral group, three animals received lesions to the left hemisphere and three animals received lesions to the right hemisphere. Contralateral lesioned animals $(n=8)$ received unilateral dorsal DG and unilateral CA3 lesions (i.e., DG was lesioned in one hemisphere, whereas CA3 was lesioned in the other hemisphere). Within this contralateral group, four animals received DG left/CA3 right lesions and four animals received DG right/CA3 left lesions.

Each animal was deeply anesthetized with isoflurane and then injected with atropine sulfate $(0.2 \mathrm{mg} / \mathrm{kg}$, intraperitoneally). The animal was placed in a stereotaxic instrument (Kopf Instruments), and an incision was made along the midline of the scalp. The skull was exposed, and the instrument was adjusted to ensure a flat skull surface. Small burr holes were drilled through the skull, and injections of neurotoxins were made through these holes. For DG lesions, injections of $0.80 \mu \mathrm{l} / \mathrm{site}\left(1.5 \times 10^{-3} \mathrm{mg} /\right.$ site at $16.00 \mu \mathrm{l} / \mathrm{hr}$ ) were made at the following coordinates: (1) $2.7 \mathrm{~mm}$ posterior to bregma, $2.1 \mathrm{~mm}$ lateral to midline, and 3.4 $\mathrm{mm}$ ventral from dura; and (2) $3.7 \mathrm{~mm}$ posterior to bregma, 2.3 $\mathrm{mm}$ lateral to midline, and $3.0 \mathrm{~mm}$ ventral from dura. For CA3 lesions, injections were made at $4.00 \mu \mathrm{l} / \mathrm{hr}$ at the following volumes and coordinates: (1) $0.05 \mu \mathrm{l}\left(3 \times 10^{-4} \mathrm{mg} / \mathrm{site}\right)$ injected at $2.5 \mathrm{~mm}$ posterior to bregma, $2.6 \mathrm{~mm}$ lateral to midline, and 3.2 $\mathrm{mm}$ ventral from dura; (2) $0.08 \mu \mathrm{l}\left(4.8 \times 10^{-4} \mathrm{mg} / \mathrm{site}\right)$ injected at $3.3 \mathrm{~mm}$ posterior to bregma, $3.3 \mathrm{~mm}$ lateral to midline, and 3.2 $\mathrm{mm}$ ventral from dura; and (3) $0.15 \mu \mathrm{l}\left(9 \times 10^{-4} \mathrm{mg} / \mathrm{site}\right)$ injected at $4.1 \mathrm{~mm}$ posterior to bregma, $4.2 \mathrm{~mm}$ lateral to midline, and $3.1 \mathrm{~mm}$ ventral from dura. All injections were made using an injection needle (28G) connected to a $10-\mu$ l Hamilton syringe with a microinjection pump (Cole Parmer Instrument Company). Previous research (Gilbert et al. 2001) using similar lesion methods for DG and CA3 have indicated that relatively specific lesions for each subregion can be made. Particular care was taken to prevent clogging of the injection needle by lowering the needle into the brain, raising the needle out from the brain, and running the injection pump to assure that the injection needle was not clogged with tissue. Once it was apparent that the needle was clear of any debris, it was again lowered to the specified coordinate, and the actual injection was made. All protocols conformed to the NIH Guide for the Care and Use of Laboratory Animals and the Institutional Animal Care and Use Committee (IACUC) at the University of Utah.

\section{Behavioral apparatus and training}

The original Hebb-Williams maze (Rabinovitch and Rosvold 1951) was modified to facilitate spatial learning. The base was painted gray and measured $76.2 \times 76.2 \mathrm{~cm}$. The walls were 30.5 $\mathrm{cm}$ high, made of 0.6-cm-thick Plexiglas. Four identical boxes, two of which served as a start box and a goal box, were placed at opposite corners of the maze. The inner barriers were made of 1.3-cm-thick Plexiglas, measuring $25.4 \mathrm{~cm}$ in height. A 7.6-cmwide black strip was placed on the bottom of the Plexiglas. This spatial arrangement was meant to facilitate the rats' use of extramaze visual cues, which included posters and three-dimensional objects surrounding the maze.

Rats were handled for about $5 \mathrm{~d}$, until they freely ate a food reward (Froot Loops cereal, Kellogg Co.) while handled, after which they were pre-trained. To prevent the rats from learning spatial cues in the testing room, pre-training was conducted in a different room, on a different behavioral apparatus (i.e., wooden linear runway). The runway was $50.8 \mathrm{~cm}$ long, with walls $10.2 \mathrm{~cm}$ high, and an open top. On each end of the runway, there was a wooden box connected to the track-one used for a start box and the other for a goal box. Each rat was placed in the start box and the door was opened to provide access to a food reward in the goal box. Once rats learned to run to the goal box to obtain a food reward in response to the opening of the start box door, testing began on the modified Hebb-Williams maze.

Rats were tested on the modified Hebb-Williams maze for six consecutive days with a fixed configuration of inner barriers. Doors for all the boxes were open except the one for the start box that was to be opened at the beginning of a trial. Only the goal box had a food reward (i.e., one full Froot Loop). The objective of each trial was to find the shortest path from the start box to the goal box, which contained a food reward. There were 11 error zones, and when the rat crossed an error zone in a specified direction, an error was recorded. As the rat learned the task, it eliminated navigational errors that deviated from the shortest path to the goal box. As soon as the rat entered the goal box, the door was closed. An intertrial interval (i.e., $30 \mathrm{sec}$ ) was given before the next trial, during which the goal box and the start box were switched. Hence, the goal box of the previous trial became the start box of the next trial. Ten trials were conducted per day for each rat. The total number of error crossings was recorded as the dependent measure for each trial.

\section{Histology}

At the conclusion of testing, each rat was deeply anesthetized with an intraperitoneal injection of sodium pentobarbital (100$200 \mathrm{mg} / \mathrm{kg}$ ). Rats were perfused intracardially with normal saline for $5 \mathrm{~min}$, followed by $10 \%$ formalin in phosphate buffered solution for $5 \mathrm{~min}$. The brain was removed from the skull and stored at $4^{\circ} \mathrm{C}$ in a solution of $10 \%$ formalin/30\% sucrose in PBS. A tissue block was cut perpendicularly from each brain. The block was frozen to about $-18^{\circ} \mathrm{C}$, then $40-\mu \mathrm{m}$ sections were cut on a Minotome cryostat (International Equipment Co.), and every third section was collected on a gelatinized, glass slide (the surface-to-surface distance between collected sections was $120 \mu \mathrm{m}$ ). The sections were stained with cresyl violet. A program, ImageJ 1.33 (National Institutes of Health, 2005), was used to quantify the extent of the lesion from $1.8 \mathrm{~mm}$ posterior to bregma to 4.3 $\mathrm{mm}$ posterior to bregma after the atlas of Paxinos and Watson (1998).

\section{Data analysis}

Mean number of errors per trial were grouped in five-trial blocks across a total of $3 \mathrm{~d}$. For a detailed analysis of the encoding and retrieval, a separate index was developed for each process. It is assumed that encoding and retrieval processes interact on a trialby-trial basis in any behavioral task. Thus, it is difficult to discriminate between the contributions of encoding and retrieval for individual trials using a behavioral analysis. On the modified Hebb-Williams maze, however, the control group exhibited measurably improved performance for Days 1 and 2, compared with the experimental group. Therefore, "encoding" and "retrieval" were given operational definitions based on calculations of trial errors. Encoding index was calculated within a day by subtracting the average of the last five trials of Day 1 (D1b) from the average of the first five trials of Day 1 (D1a). Retrieval index was calculated by subtracting the average of the first five trials of Day 2 (D2a) from the average of the last five trials of Day 1 (D1b). Therefore, a larger encoding score (D1a - D1b) indicated more effective encoding during Day 1, and a larger retrieval score (D1b - D2a) indicated more effective retrieval between Days 1 and 2 . The assumption made in this study was that the encoding of information predominated during testing on Day 1 and the retrieval of information predominated during testing on Day 2, $24 \mathrm{~h}$ later.

\section{Acknowledgments}

This research was supported by NIH 5R01MH065314-02 and NSF IBN-0135273 awarded to R.P. Kesner.

\section{References}

Gilbert, P.E. and Kesner, R.P. 2003. Localization of function within the hippocampus: The role of the dorsal CA3 subregion in paired-associate learning. Behav. Neurosci. 117: 1385-1394. 
.2006. The role of the dorsal CA3 hippocampal subregion in spatial working memory and pattern separation. Behav. Brain Res. 169: $142-149$.

Gilbert, P.E., Kesner, R.P., and Lee, I. 2001. Dissociating hippocampal subregions: A double dissociation between the dentate gyrus and CA1. Hippocampus 11: 626-636.

Goldschmidt, R.B. and Steward, O. 1982. Neurotoxic effects of colchicine: Differential susceptibility of CNS neuronal populations. Neuroscience 7: 695-714.

Goodrich-Hunsaker, N.J., Hunsaker, M.R., and Kesner, R.P. 2005. Effects of hippocampus sub-regional lesions for metric and topological spatial information processing. Abstract Viewer/Itinerary Planner Program No. 647.1. Society for Neuroscience, Washington, D.C.

Jerman, T.S., Kesner, R.P., Lee, I., and Berman, R.F. 2005. Patterns of cell loss based on subregional lesions of the hippocampus. Brain Res. 1065: $1-7$

Kesner, R.P., Lee, I., and Gilbert, P. 2004. A behavioral assessment of hippocampal function based on a subregional analysis. Rev. Neurosci. 15: $333-351$.

Lassalle, J.M., Bataille, T., and Halley, H. 2000. Reversible inactivation of the hippocampal mossy fiber synapses in mice impairs spatial learning, but neither consolidation nor memory retrieval, in the Morris navigation task. Neurobiol. Learn. \& Mem. 73: 243-257.

Lee, I. and Kesner, R.P. 2002. Differential contribution of NMDA receptors in hippocampal subregions to spatial working memory. Nat. Neurosci. 5: 162-168.

.2003. Differential role of dorsal hippocampus subregions in spatial working memory with short versus intermediate delay. Behav. Neurosci. 117: 1044-1053.

.2004a. Differential contributions of dorsal hippocampal subregions to memory acquisition and retrieval in contextual fear-conditioning. Hippocampus 14: 301-310.

.2004b. Encoding versus retrieval of spatial memory: Double dissociation between the dentate gyrus and the perforant path inputs into CA3 in the dorsal hippocampus. Hippocampus 14: 66-76.

Leutgeb, S., Leutgeb, J.K., Treves, A., Moser, M.B., and Moser, E.I. 2004. Distinct ensemble codes in hippocampal areas CA3 and CA1. Science 305: 1295-1298.

Marr, D. 1971. Simple memory: A theory of archicortex. Phil. Trans. $R$. Soc. London B 262: 23-81.

Mundy, W.R. and Tilson, H.A. 1990. Neurochemical recovery in the neocortex after colchicine lesions of the nucleus basalis magnocellularis in rats. Brain Res. Bull. 25: 207-209.

Nakazawa, K., Sun, L.D., Quirk, M.C., Rondi-Reig, L., Wilson, M.A., and Tonegawa, S. 2003. Hippocampal CA3 NMDA receptors are crucial for memory acquisition of one-time experience. Neuron 38: 305-315.

Nanry, K.P., Mundy, W.R., and Tilson, H.A. 1989. Colchicine-induced alternations of reference memory in rats: Role of spatial versus non-spatial task components. Behav. Brain Res. 35: 45-53.

Paxinos, G. and Watson, C. 1998. The rat brain in stereotaxic coordinates. Academic Press, San Diego, CA.

Poucet, B. 1989. Object exploration, habituation, and response to a spatial change in rats following septal or medial frontal cortical damage. Behav. Neurosci. 103: 1009-1016.

Rabinovitch, M.S. and Rosvold, H.E. 1951. A closed-field intelligence test for rats. Can. J. Psychol. 5: 122-128.

Rolls, E.T. 1996. A theory of hippocampal function in memory. Hippocampus 6: 601-620.

Sutherland, R.J., Whishaw, I.Q., and Kolb, B. 1983. A behavioural analysis of spatial localization following electrolytic, kainate- or colchicine-induced damage to the hippocampal formation in the rat. Behav. Brain Res. 7: 133-153.

Tanila, H. 1999. Hippocampal place cells can develop distinct representations of two visually identical environments. Hippocampus 9: $235-246$.

Vago, D.R. and Kesner, R.P. 2005. An electrophysiological and behavioral characterization of the temporoammonic pathway: Disruption produces deficits in retrieval and spatial mismatch. Abstract Viewer/Itinerary Planner, Program No. 647.5. Society for Neuroscience, Washington, D.C.

Vazdarjanova, A. and Guzowski, J.F. 2004. Differences in hippocampal neuronal population responses to modifications of an environmental context: Evidence for distinct, yet complementary, functions of CA3 and CA1 ensembles. J. Neurosci. 24: 6489-6496.

Xavier, G.F., Oliveira-Filho, F.J.B., and Santos, A.M.G. 1999. Dentate gyrus-selective colchicine lesion and disruption of performance in spatial tasks: Difficulties in "place strategy" because of a lack of flexibility in the use of environmental cues? Hippocampus 9: $668-681$.

Received March 9, 2006; accepted in revised form May 30, 2006.

\section{Learning \& Memory}

www.learnmem.org 


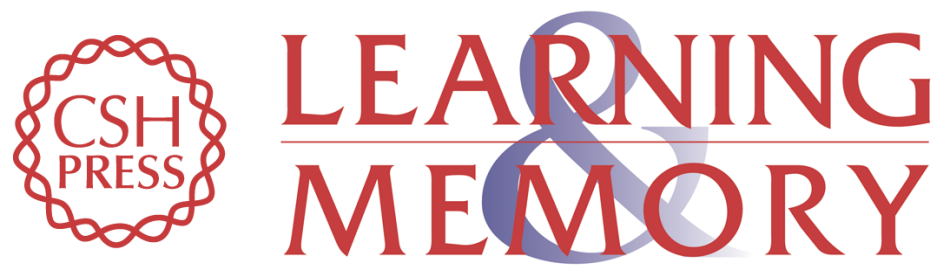

\section{Disconnection analysis of CA3 and DG in mediating encoding but not retrieval in a spatial maze learning task}

Taylor Jerman, Raymond P. Kesner and Michael R. Hunsaker

Learn. Mem. 2006, 13:

Access the most recent version at doi:10.1101//m.246906

References This article cites 23 articles, 2 of which can be accessed free at: http://learnmem.cshlp.org/content/13/4/458.full.html\#ref-list-1

License

Email Alerting Receive free email alerts when new articles cite this article - sign up in the box at the Service top right corner of the article or click here. 